\title{
Recurrent Neural Network Based Simulation of a Single Shaft Gas Turbine
}

\author{
Hamid Asgari $^{1} \quad$ Emmanuel Ory $^{1} \quad$ Jari Lappalainen $^{2}$ \\ ${ }^{1}$ VTT Technical Research Centre of Finland Ltd., Espoo, Finland, \\ \{hamid.asgari, emmanuel.ory\}dvtt.fi \\ ${ }^{2}$ Semantum Oy, Finland, \\ jari.lappalainen@semantum.fi
}

\begin{abstract}
In this study, a model of a single shaft gas turbine (GT) is developed by using artificial intelligence (AI). A recurrent neural network $(R N N)$ is employed to train the datasets of the GT variables in Python programming environment by using Pyrenn Toolbox. The resulting model is validated against the Test datasets. Thirteen significant variables of the gas turbine are considered for the modelling process. The results show that the $R N N$ model developed in this study is capable of performance prediction of the system with a high reliability and accuracy. This methodology provides a simple and effective approach in dynamic simulation of gas turbines, especially when real datasets are only available over a limited operational range and using simulated datasets for modelling and simulation purposes is unavoidable.
\end{abstract}

Keywords: gas turbine, modelling, simulation, artificial intelligence, recurrent neural network, black-box model

\section{Introduction}

Todays, Artificial intelligence (AI) plays a key role in the industrial world and has a meaningful and influential presence in many aspects of daily life. $A I$ is an area of computer science that deals with performing human-like tasks. As one of the most popular applications of $A I$, one can refer to Machine learning (ML). $M L$ is a research area that deals with data analysis, and a variety of approaches for computers to learn from datasets when no specific algorithm exits. $M L$ is used in many industrial systems such as gas turbines for pattern recognition, system identification, trouble shooting, sensor validation, condition monitoring, modelling, simulation, and performance prediction (Liu et al., 2020; Cisotto and Herzallah, 2018; Kiakojoori and Khorasani, 2016).

One of the main tools used in machine learning is artificial neural network (ANN). ANN, as a subset of artificial intelligence, has been widely used over the past decades. The main idea for creating an $A N N$ was to mimic the human brain as a simple model that was capable of solving complex problems in different scientific fields. As a brain-inspired data-driven model, $A N N$ has shown to be a very good alternative to whitebox approach for modelling and simulation of industrial systems and processes. It has been very useful for the researchers whose activities cover a wide range of system identification and simulation techniques. A recurrent neural network is a subset of $A N N$ that is used to setup dynamic models of industrial systems.

Gas turbines are broadly employed, such as airplane engines. They are also widely used for power generation, especially in the areas that are far away from cities; where access to the national electrical grid is limited. Oil \& gas fields, offshore platforms, ships, and marine infrastructures are examples of such places. The significant role of $G T \mathrm{~s}$ in industry has strongly encouraged and motivated researchers to investigate new methodologies for modelling these complex systems. The desired models should be able to capture the system dynamics as accurately as possible. So far, many different experimental and analytical methodologies have been explored to reach this goal. The research in this area is included in both stationary (mainly located in power plants) and aero gas turbine engines (Asgari et al., 2012, 2013a).

$M L$-based techniques like $A N N$ have shown the capability to predict dynamic behavior of GTs without having access to information about the system physics. Different ANN-based methodologies have already been investigated and developed in order to disclose complex nonlinear behavior of aero gas turbines (Agrawal and Yunis, 1982; Chiras et al., 2001, 2002; Ruano et al., 2003; Torella et al., 2003; Sarkar et al., 2012, 2013; Salehi and Montazeri, 2018; Ibrahem et al. 2019). These efforts have covered a variety of approaches such as $M L P$ (multi-layer perceptron), NARMAX (nonlinear auto-regressive moving average with exogenous inputs), NARX (nonlinear autoregressive exogenous model), RBF (radial basis function), BPNN (back propagation neural networks), and BSNN (B-spline neural networks).

Besides, many studies have focused on ANN-based modelling of industrial power plant gas turbines $(I P G T)$. The results of these studies demonstrated the capability of $A N N$ in capturing IPGT dynamics 
(Lazzaretto and Toffolo, 2001; Kim et al., 2001, 2002; Ogaji et al., 2002; Basso et al., 2004; Jurado, 2005; Simani and Patton, 2008; Fast et al., 2008, 2009; Yoru et al., 2009; Tavakoli et al., 2009; Fast and Palmé, 2010; Palmé et al., 2011; Bartolini et al., 2011; Asgari et al., 2013b, 2014, 2015, 2016). ANN has also been used for control-oriented modelling of GTs (Asgari et al., 2017). $A N N$ may be used for fault identification and warning generation with high reliability. Arriagada et al. (2003), Elashmawi et al. (2017, 2018), and Rahmoune et al. (2017) used novel ANN-based models for monitoring and fault detection of industrial gas turbines.

From the literature survey, many efforts have been made in the area of black-box modelling of gas turbines, with their own advantages and limitations. However, because of complex nonlinear dynamics of GTs, and their different applications, sizes and capacities, study in this area is still a challenging issue. Further research still need to be carried out to develop the GT models, and to optimize design and performance of these engines. The work reported in this publication can help to disclose details of the problems that cause unpredictable shutdowns, over-heating, and over-speed of GTs on industrial sites.

In this study, a black-box model of a single shaft gas turbine is modelled and simulated by using a recurrent neural network. The $R N N$ is employed to train datasets of the gas turbine in Python programming environment. First, the performance of a typical gas turbine is briefly explained in Section 2. Then, in Section 3, a short description about recurrent neural networks is presented. Section 4 describes modelling procedure including data acquisition, data processing, building $R N N$ structure, code generation, and training process. The results are presented in Section 5. Finally, the output of the research is briefly discussed in Section 6 .

\section{Gas Turbines}

A gas turbine is classified as an internal combustion engine that converts chemical energy to mechanical energy. It is widely used for industrial applications. $G T$ may be connected to a generator, pump or compressor as the main driver to shape a turbo-generator, a turbopump, or a turbo-compressor respectively. Figure 1 illustrates the main components of a single shaft opencycle constant-pressure gas turbine engine (Encyclopedia Britannica, Inc., 1999). Both the compressor and the turbine are installed on the central shaft and rotate together.

Operation of an industrial gas turbine is shown in Figure 2. According to this figure, the compressor receives air at point 1 and delivers hot compressed air to the combustion chamber (combustor) at point 2 . The mixture of fuel and air is ignited inside the combustion chamber to form the hot gases at point 3 . These hot gases pass through the turbine and rotate it. The output power of the turbine provides the required energy for driving the compressor part, and the GT mechanical output.

Operation of a gas turbine is based on Brayton cycle. A temperature-entropy (T-S) framework-based of a standard Brayton cycle is illustrated in Figure 3 (Arabi et al., 2019). According to this figure, 1-2-3-4-1 shows the ideal cycle, while $1-2^{\prime}-3^{\prime}-4^{\prime}-1$ indicates the real cycle. In the real cycle, processes in both the compressor $\left(1-2^{\prime}\right)$ and the turbine $\left(3^{\prime}-4^{\prime}\right)$ are non-isentropic and irreversible. In the ideal cycle, these processes are assumed isentropic. Processes 2-3 and 4-1 may be considered isobar, if pressure losses in combustor and air filters are neglected (Walsh and Fletcher, 1998).

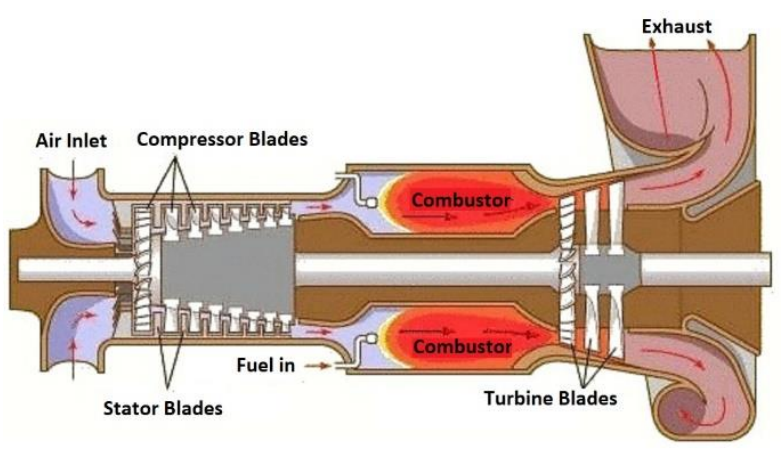

Figure 1. A typical single shaft gas turbine engine (Encyclopedia Britannica, Inc., 1999).

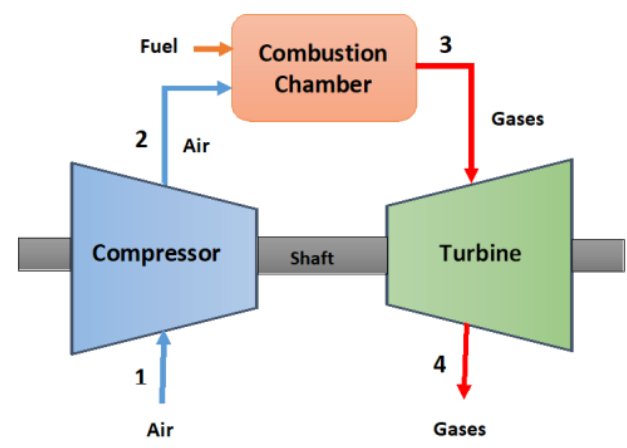

Figure 2. A schematic of a typical single shaft gas turbine.

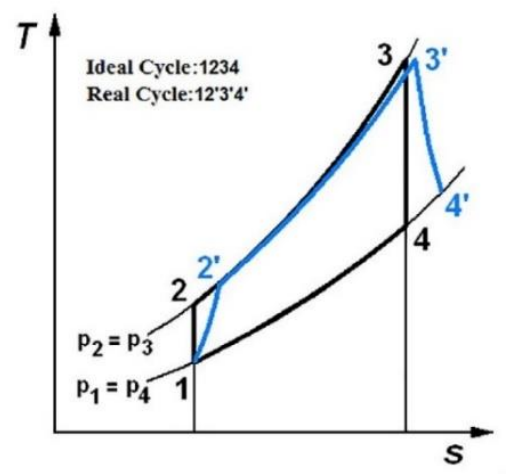

Figure 3. Ideal and real Brayton cycles in temperatureentropy diagram (Arabi et al., 2019). 


\section{Recurrent Neural Networks}

Simulation of all industrial systems can be carried out by using white-box or black-box models. When knowledge and information about the physics involved in a system are quite enough and dynamic model equations are available, white-box models can be employed, and different simulation software such as $M A T L A B$ and APROS may be used for modelling of the system dynamics. MATLAB is a multi-functional programming platform designed and developed by MathWorks for numerical computing in engineering and scientific applications. APROS is also a multi-functional software for modelling and dynamic simulation of industrial processes, such as power plants and other energy systems (Fortum and VTT, 2020). Dynamic equations usually need to be simplified (e.g. linearized) because of their nonlinear and coupled natures. However, the simplification process may negatively affect the accuracy of the resulting model.

When the physics of a system is unknown or access to the relevant knowledge, dynamic equations and efficient solution techniques is limited, black-box modelling approach can be a good choice, and sometimes unavoidable. Black-box models can disclose the relationships among the system variables by employing input and output datasets. These datasets can be simulated or measured (experimental) data. Simulated data may be used when operational data are not available.

$A N N$ is a class of black-box models that can be used for simulation of gas turbines and other industrial equipment (Tiumentsev and Egorchev, 2019). The structure of an $A N N$ consists of input, hidden and output layers. Each $A N N$ may have more than one hidden layer. The layers may have different number of artificial neurons. These neurons (units) are internally connected by transfer functions, which can be linear or nonlinear. Artificial neural networks are capable of learning the relationship among inputs and outputs of a system through an iterative training process. Each input into the neuron is associated with its own adjustable number, which is called weight. Weights are determined during the training process. The complexity of a system dynamics determines the number of hidden layers and their associated neurons. Figure 4 illustrates a typical $A N N$ including three inputs, two outputs and one hidden layer with four neurons.

Among different $A N N$ algorithms for static and dynamic modellings, $R N N$ can be employed for the modelling of dynamic industrial systems. In a recurrent neural network, each layer has a recurrent connection. This enables $R N N$ to propagate data forward and backward, from later processing stages to earlier ones, allowing the network to have an infinite dynamic response to the input data. As a universal approximator, $R N N$ has shown excellent dynamic ability to deal with various input and output types for modelling and simulation of industrial systems.

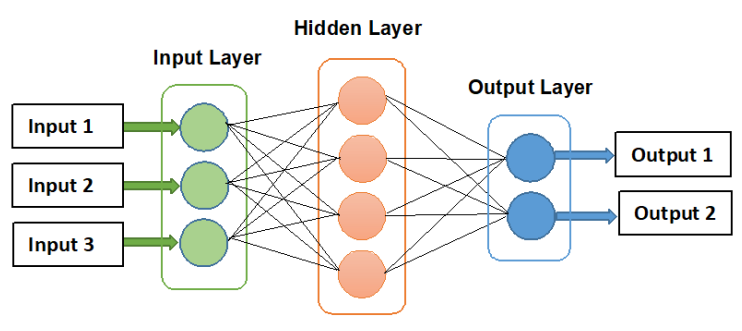

Figure 4. The structure of a typical artificial neural network with input, hidden and output layers.

\section{Modelling Procedure}

To approach an $R N N$ model with a high accuracy for the gas turbine of this study, a variety of structures was considered. These structures were set up based on the data type, training algorithms, types of activation functions, number of hidden layers, number of neurons, and values of the weights and biases. The goal was to attain a structure with the high capability of accurate prediction of the $G T$ dynamic behavior. Using the most effective $G T$ variables as inputs and outputs is vital for building a reliable model. Data availability, system knowledge, and modelling objectives are fundamental factors that should be considered for selection of the $R N N$ inputs and outputs.

\subsection{Data Acquisition}

The first step in $R N N$ modelling is to obtain enough reliable datasets. A dynamic model of a low-power gas turbine, simulated in Simulink/MATLAB environment was employed for data generation (Asgari et al. 2013b). The relevant white-box model was already developed and verified against experimental datasets (Ailer et al., 2002). Totally, 3000 datasets were generated for 13 different $G T$ variables. In this study, the purpose of a dataset is a vector with 13 single values, corresponding to 13 different $G T$ variables. Therefore, 3000 datasets cover 3000 vectors like that.

The obtained datasets were categorized as inputs and outputs of the gas turbine engine according to Table 1 and Table 2 respectively. As it can be seen from the tables, fuel rate, ambient temperature, ambient pressure, and load were determined as four main gas turbine inputs, while temperature and pressure at different operational points (corresponding to the numbers in Figures 2 and 3), compressor pressure ratio, rotational speed, and gas turbine efficiency were considered as GT outputs. To be able to evaluate the model generalization, and to avoid over-fitting during the training process, the datasets were alternatively divided into the Train and Test groups. Half of the datasets (1500 out of 3000) were employed for training the $R N N$, and the remaining datasets were used to test the resulting model. 
Table 1. Gas Turbine Input Parameters.

\begin{tabular}{|l|c|c|c|}
\hline \multicolumn{1}{|c|}{ Variable } & Symbol & Unit & $\begin{array}{c}\text { Operational } \\
\text { Range }\end{array}$ \\
\hline Fuel rate & $M_{f}$ & $\mathrm{~kg} / \mathrm{s}$ & {$[0.00367 ; 0.027]$} \\
\hline $\begin{array}{l}\text { Ambient } \\
\text { temperature }\end{array}$ & $T_{01}$ & $\mathrm{~K}$ & {$[243.15 ; 308.15]$} \\
\hline $\begin{array}{l}\text { Ambient } \\
\text { pressure }\end{array}$ & $P_{01}$ & $\mathrm{kPa}$ & {$[60 ; 110]$} \\
\hline Load & $M_{\text {Load }}$ & $\mathrm{N} . \mathrm{m}$ & {$[0 ; 363]$} \\
\hline
\end{tabular}

Table 2. Gas Turbine Output Parameters.

\begin{tabular}{|l|c|c|}
\hline \multicolumn{1}{|c|}{ Variable } & Symbol & Unit \\
\hline Temperature at point 2 & $T_{02}$ & $\mathrm{~K}$ \\
\hline Temperature at point 3 & $T_{03}$ & $\mathrm{~K}$ \\
\hline Temperature at point 4 & $T_{04}$ & $\mathrm{~K}$ \\
\hline Pressure at point 2 & $P_{02}$ & $\mathrm{kPa}$ \\
\hline $\begin{array}{l}\text { Pressure at point } 3 \\
\text { Rotational speed } \\
\text { (number of revolutions) }\end{array}$ & $P_{03}$ & $\mathrm{kPa}$ \\
\hline Gas turbine efficiency & $P_{04}$ & $\mathrm{kPa}$ \\
\hline Pressure at point 4 & $C P R$ & - \\
\hline Compressor pressure ratio & $E_{G T}$ & - \\
\hline
\end{tabular}

\subsection{RNN Structure}

Since the gas turbine is a dynamic system, the aim of this research is to create a recurrent neural network, in which the output parameters of the current time-step depends on the output parameters in the previous one. In this study, an $R N N$ model was designed with four inputs, one hidden layer, and nine outputs. The network was named $R N N 4-H-9$ according to its structure (see Figure 5).

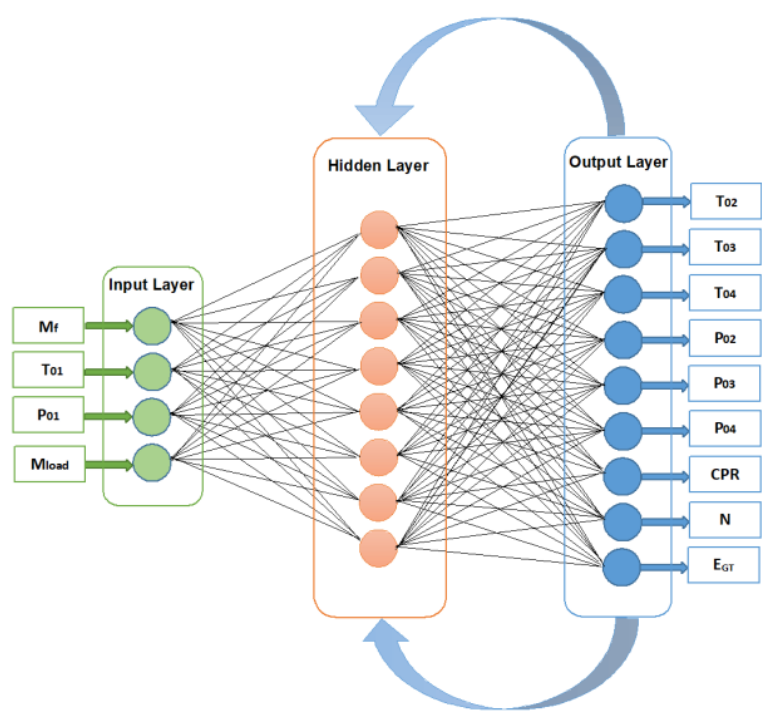

Figure 5. The structure of the recurrent neural network RNN 4-H-9.

\subsection{Code Generation \& Training Process}

To achieve an $R N N$ model with good generalization characteristic, details of the network structure should be determined as accurately as possible. For this purpose, a comprehensive computer code was developed in Python programming environment. Python is a high level, interpreted, and object-oriented programming language, firstly created and used by Guido van Rossum in 1991. The Pyrenn Toolbox, integrated in Python, was used for training and testing RNN 4-H-9. Pyrenn allows the creation of a wide range of $R N N$ configurations. It employs LM (Levenberg-Marquardt) algorithm for training the network. $L M$ is a second-order QuasiNewton optimization algorithm, which is much faster than first-order methods such as gradient descent (Suzuki, 2011). The RNN 4-H-9 model was trained by following a flexible programming code and assigning a combination of different values for the number of neurons in the hidden layer, the maximum number of iterations, the number of recurrent connections, and delay in the recurrent connections. The results of the simulation were figured for all the nine $G T$ output parameters, and were compared to both the Train and Test datasets. The goal was to see how accurately the $R N N$ outputs follow both the Train and Test datasets trends. According to the results, the optimal RNN model was achieved after 1200 iterations, with eight neurons in the hidden layer, a recurrent connection with delay of 1 time-step in the hidden layer, and a recurrent connection with delay of 1 and 2 time-steps from the output to the first layer.

The root mean squared relative error (RMSRE) of the results for the $R N N$ model was calculated according to Equation (1), where $n_{d}$ is the number of datasets, $y$ is the available (simulated) data, and $y_{m}$ is the prediction of the $R N N$ model. 


$$
\operatorname{RMSRE}(\%)=100 * \sqrt{\frac{1}{n_{d}} \sum_{i=1}^{n_{d}}\left(\frac{y-y_{m}}{y}\right)^{2}}
$$

\section{Results}

Figures 6 to 14 compare outputs of the $G T$ with the outputs of $R N N$ 4-H-9, for both the Train and Test datasets (targets). Figures 6 to 8 show the comparison between outputs of the RNN model and the GT Train and Test data for temperatures at different operational points of the $G T\left(T_{02}, T_{03}, T_{04}\right)$. In Figures 9 to 11, the results are shown for the pressure variables $\left(p_{02}, p_{03}\right.$, $p_{04}$ ). Figures 12, 13, and 14 indicate the same comparison for the compressor pressure ratio $(C P R)$, rotational speed $(N)$, and $G T$ efficiency $\left(E_{G T}\right)$ respectively. Comparisons between outputs of the $G T$ and the $R N N$ model for the Train datasets demonstrate satisfactory results for the training process. The resulting $R N N$ model was validated against the Test datasets. According to Figures 6 to 14, the validation results are also satisfactory for all GT output parameters. As these figures show, the outputs of $R N N 4-\mathrm{H}-9$ follow the targets very closely for both the Train and Test datasets.

Figure 15 shows a comparison between RMSRE (\%) of $R N N$ 4-H-9 outputs for the Train and Test datasets. According to this figure, the average RMSRE (\%) of the nine output variables (Ave.) for the Train and Test datasets are about $0.22 \%$ and $2.6 \%$ respectively. It demonstrates that the $R N N$ model developed in this study has a high reliability and accuracy in capturing the system dynamics.

\section{Conclusions}

In this study, a recurrent neural network of a single shaft gas turbine was investigated and developed in Python programming environment by using Pyrenn Toolbox, and employing 3000 simulated datasets for thirteen significant variables of the $G T$. The resulting model was validated against the Test datasets. The results demonstrated that response of the $R N N$ model to variations in input parameters followed the system outputs with an acceptable accuracy. It proves that the $R N N$ model is capable of performance prediction of the system with a high reliability. The methodology employed in this study provides a simple and reliable approach in dynamic modelling and simulation of gas turbines, especially when access to operational datasets is limited for any reason.

\section{Acknowledgements}

This work was carried out during the tenure of an ERCIM 'Alain Bensoussan' Fellowship Programme at VTT Technical Research Centre of Finland Ltd, Espoo, Finland. ERCIM stands for the European Research Consortium for Informatics and Mathematics.

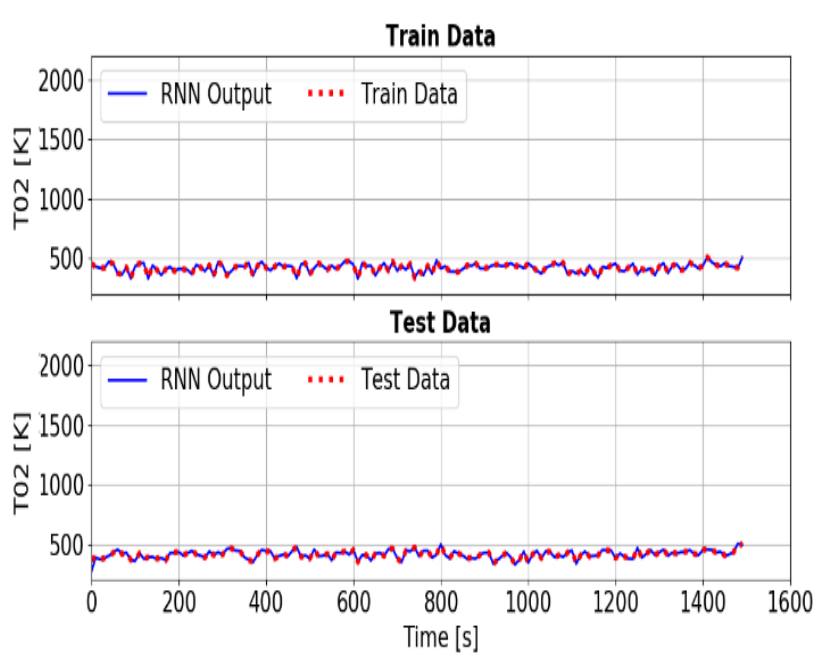

Figure 6. A comparison between output of the $R N N$ model and the GT Train and Test datasets for $T_{02}$.

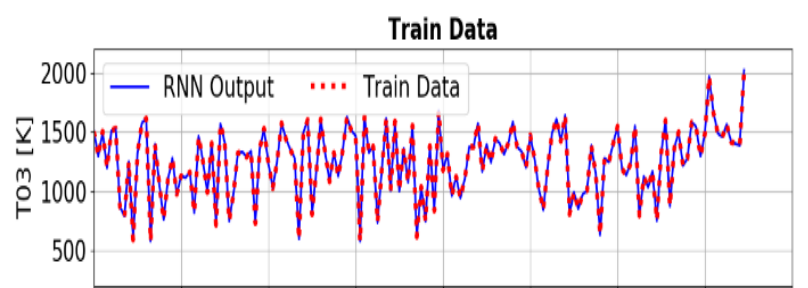

Test Data

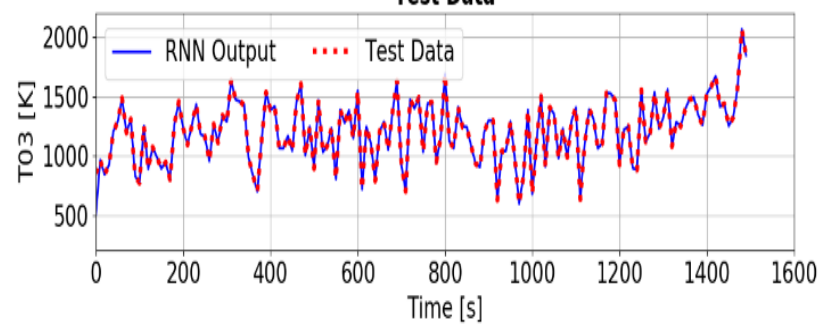

Figure 7. A comparison between output of the $R N N$ model and the GT Train and Test datasets for $T_{03}$.

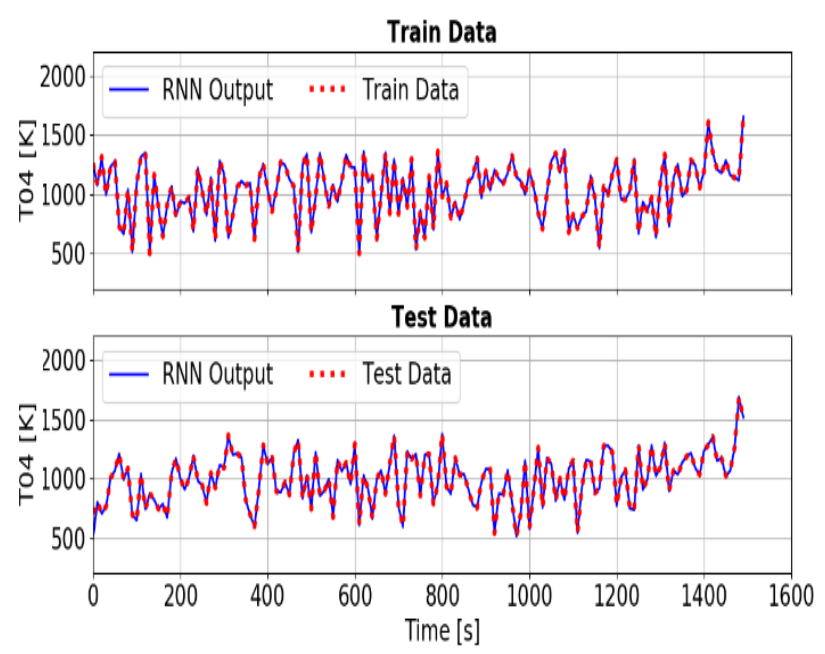

Figure 8. A comparison between output of the $R N N$ model and the GT Train and Test datasets for $T_{04}$. 

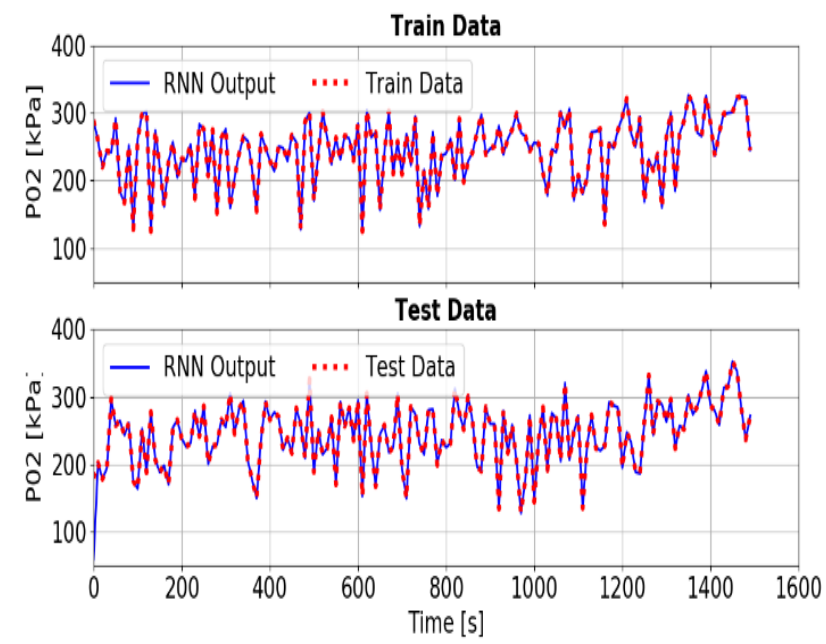

Figure 9. A comparison between output of the $R N N$ model and the GT Train and Test datasets for $P_{02}$.
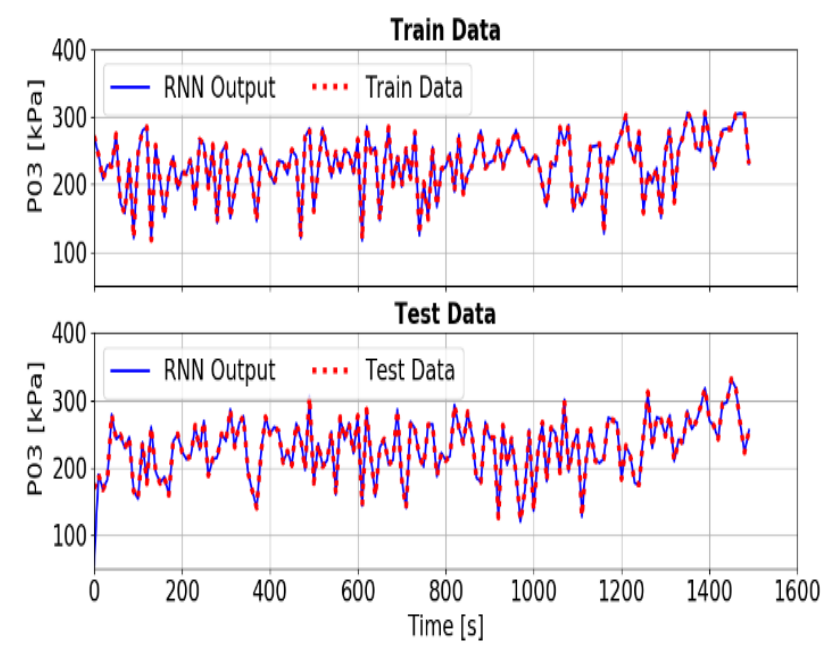

Figure 10. A comparison between output of the $R N N$ model and the GT Train and Test datasets for $P_{03}$.
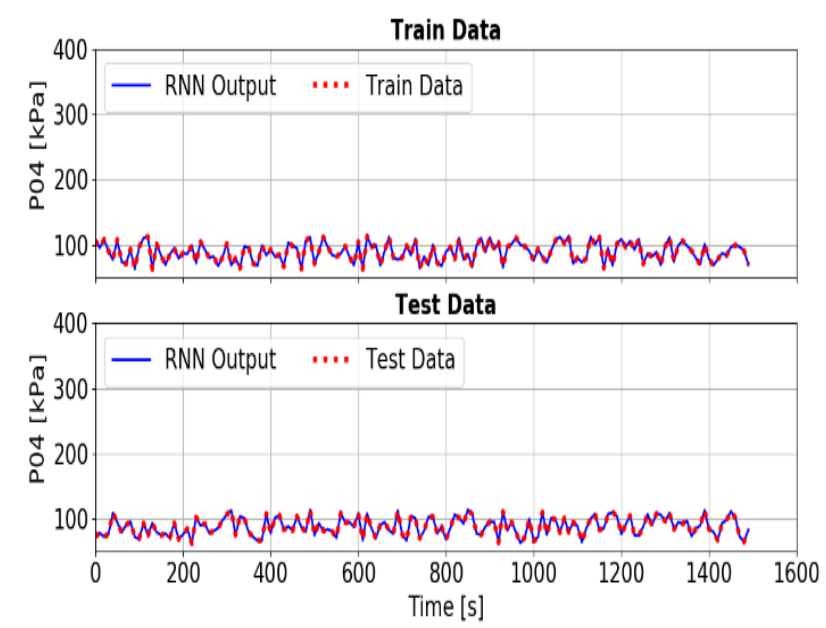

Figure 11. A comparison between output of the $R N N$ model and the GT Train and Test datasets for $P_{04}$.

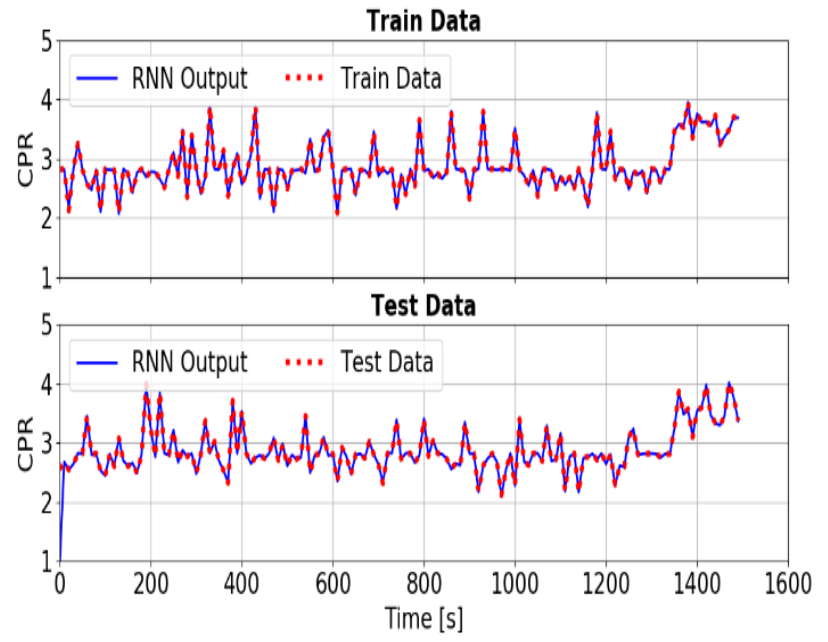

Figure 12. A comparison between output of the $R N N$ model and the GT Train and Test datasets for compressor pressure ratio $(C P R)$.
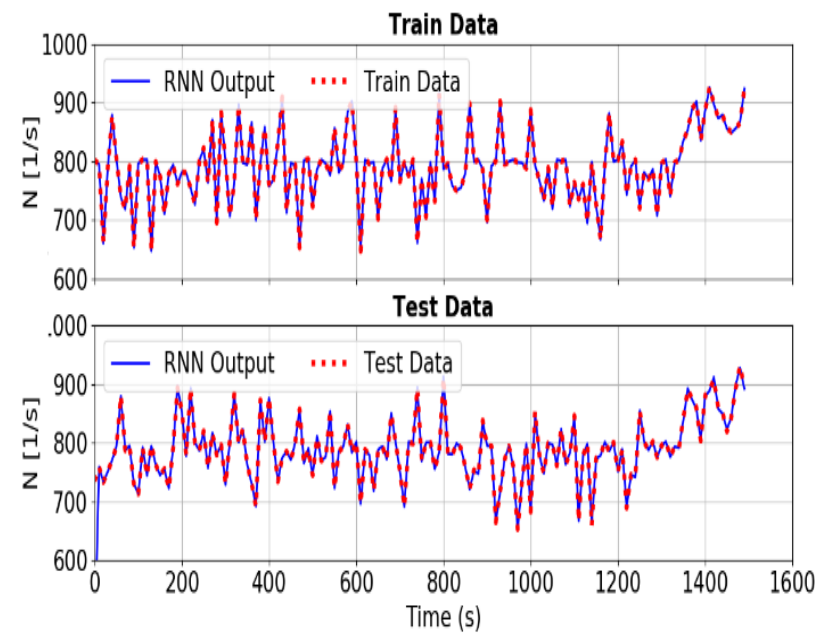

Figure 13. A comparison between output of the $R N N$ model and the GT Train and Test datasets for rotational speed $(N)$.

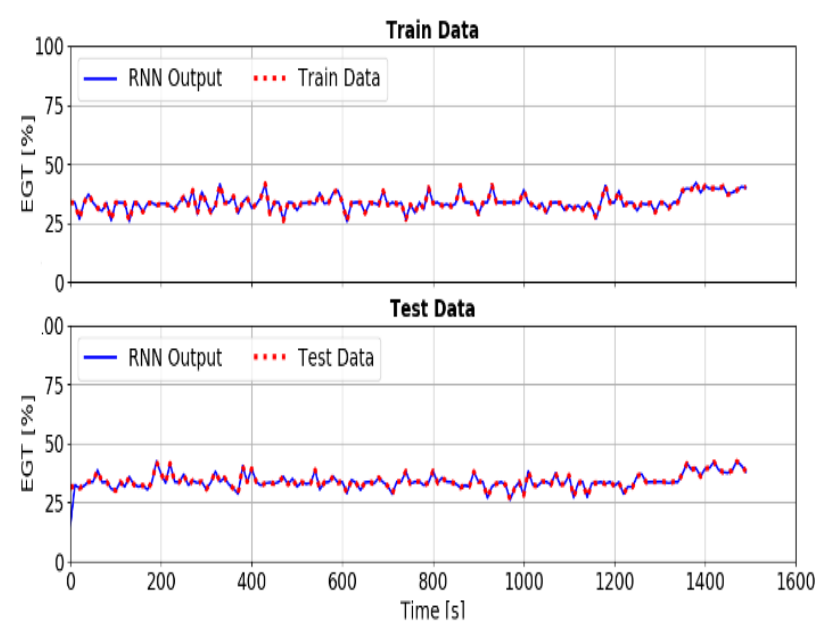

Figure 14. A comparison between output of the $R N N$ model and the GT Train and Test datasets for GT efficiency $\left(E_{G T}\right)$. 


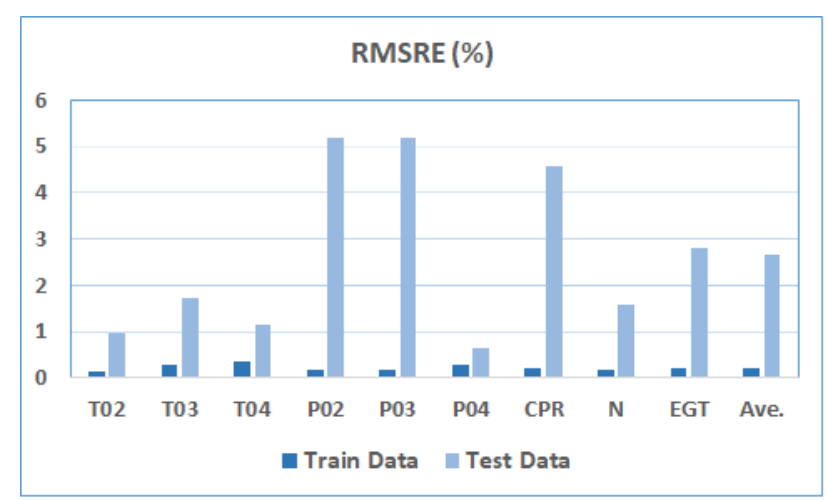

Figure 15. RMSRE (\%) of $R N N$ 4-H-9 outputs for the $G T$ Train and Test datasets.

\section{References}

R. K. Agrawal and M. Yunis. A Generalized Mathematical Model to Estimate Gas Turbine Starting Characteristics. ASME Journal of Engineering for Power, 104(1):194-201, 1982. doi:10.1115/1.3227249.

P. Ailer, I. Santa, G. Szederkenyi and K. M. Hangos. Nonlinear Model-Building of a Low-Power Gas Turbine. Periodica Polytechnica Transportation Engineering, 29(12):117-135, 2002. URL:eprints.sztaki.hu/2872.

S. M. Arabi, H. Ghadamian, M. Aminy, H. A. Ozgoli, B. Ahmadi and M. Khodsiani. The Energy Analysis of GE-F5 Gas Turbines Inlet Air-Cooling Systems by the Off-Design Method. Measurement and Control, 52(9-10):1489-1498, 2019. doi:10.1177/0020294019877485.

J. Arriagada, M. Genrup, A. Loberg and M. Assadi. Fault Diagnosis System for an Industrial Gas Turbine by Means of Neural Networks. Proceedings of the International Gas Turbine Congress, Tokyo, Japan, 2003.

H. Asgari, X. Q. Chen, M. B. Menhaj and R. Sainudiin. ANNbased System Identification, Modelling and Control of Gas Turbines - A Review. Advanced Materials Research, Vol. 622-623, pp. 611-617, 2012. doi:10.4028/www.scientific.net/amr.622-623.611.

H. Asgari, X. Q. Chen and R. Sainudiin. Modelling and Simulation of Gas Turbines. International Journal of Modelling, Identification, and Control (IJMIC), 20(3):253270, 2013a. doi:10.1504/ijmic.2013.057137.

H. Asgari, X. Q. Chen, M. B. Menhaj and R. Sainudiin. Artificial Neural Network Based System Identification for a Single-Shaft Gas Turbine. ASME Journal of Engineering for Gas Turbines and Power, 135(9):092601 (7 pages), 2013b. doi:10.1115/1.4024735.

H. Asgari, X. Q. Chen, R. Sainudiin, M. Morini, M. Pinelli, P. R. Spina and M. Venturini. Modelling and Simulation of the Start-Up Operation of a Heavy-Duty Gas Turbine Using NARX Models. Proceedings of ASME Turbo Expo 2014, Vol. 3A, GT2014-25056, 10 pages, Düsseldorf, Germany, 2014. doi:10.1115/gt2014-25056.

H. Asgari. Modelling, Simulation, and Control of Gas Turbines Using Artificial Neural Networks. PhD Thesis, University of Canterbury, Christchurch, New Zealand, 230 pages, 2014. URL:http://hdl.handle.net/10092/9355.
H. Asgari, M. Venturini, X. Q. Chen and R. Sainudiin. Modeling and Simulation of the Transient Behavior of an Industrial Power Plant Gas Turbine. ASME Journal of Engineering for Gas Turbines and Power, 136(6):061601 (10 pages), 2014. doi:10.1115/1.4026215.

H. Asgari and X. Q. Chen. Gas Turbines Modelling, Simulation, and Control: Using Artificial Neural Networks. Taylor \& Francis, CRC Press, USA, 206 pages, ISBN 9781138893443, 2015. doi:10.1201/b18956.

H. Asgari, X. Q. Chen, M. Morini, M. Pinelli, R. Sainudiin, P. R. Spina and M. Venturini. NARX Models for Simulation of the Start-Up Operation of a Single-Shaft Gas Turbine. Applied Thermal Engineering, Vol. 93, pp. 368-376, 2016. doi:10.1016/j.applthermaleng.2015.09.074.

H. Asgari, M. Fathi and X. Q. Chen. Design of Conventional and Neural Network Based Controllers for a Single-Shaft Gas Turbine. Aircraft Engineering and Aerospace Technology, Emerald Publishing Limited, 89(1):52-65, 2017. doi:10.1108/aeat-11-2014-0187.

M. R. B. Tavakoli, B. Vahidi and W. Gawlik. An Educational Guide to Extract the Parameters of Heavy-Duty Gas Turbines Model in Dynamic Studies Based on Operational Data. IEEE Transactions on Power Systems, 4(3):13661374, 2009. doi:10.1109/tpwrs.2009.2021231.

C. M. Bartolini, F. Caresana, G. Comodi, L. Pelagalli, M. Renzi and S. Vagni. Application of Artificial Neural Networks to Micro Gas Turbines. Energy Conversion and Management, 52(1):781-788, 2011. doi:10.1016/j.enconman.2010.08.003

M. Basso, L. Giarre, S. Groppi and G. Zappa. NARX Models of an Industrial Power Plant Gas Turbine. IEEE Transactions on Control Systems Technology, 13(4):599604, 2004. doi:10.1109/tcst.2004.843129.

M. B. Rahmoune, A. Hafaifa and G. Mouloud. Fault Diagnosis in Gas Turbine Based on Neural Networks: Vibrations Speed Application. Advances in Acoustics and Vibration, Springer, Vol. 5, pp. 1-11, 2017. doi:10.1007/978-3-319-41459-1_1.

N. Chiras, C. Evans and D. Rees. Nonlinear Modelling and Validation of an Aircraft Gas Turbine Engine. Nonlinear Control Systems, 34(6):871-876, IFAC Symposia Series, 2001. doi:10.1016/s1474-6670(17)35289-8.

N. Chiras, C. Evans and D. Rees. Nonlinear Gas Turbine Modelling Using Feedforward Neural Networks. Proceedings of ASME Turbo Expo 2002, Vol. 2, GT200230035, pp. 145-152, Amsterdam, Netherlands, 2002. doi:10.1115/gt2002-30035.

N. Chiras, C. Evans and D. Rees. Global Nonlinear Modelling of Gas Turbine Dynamics Using NARMAX Structures. ASME Journal of Engineering for Gas Turbines and Power, 124(4):817-826, 2002. doi:10.1115/1.1470483.

S. Cisottoy and R. Herzallah. Performance Prediction Using Neural Network and Confidence Intervals: A Gas Turbine Application. Proceedings of 2018 IEEE International Conference on Big Data, pp. 2151-2159, Seattle, WA, USA, 2018. doi:10.1109/BigData.2018.8621919.

W. H. Elashmawi, N. A. Kotp and G. E. Tawel. Neural Network Monitoring Model for Industrial Gas Turbine. Asian Journal of Applied Sciences, 5(3):608-614, 2017. URL:http://orcid.org/0000-0003-0142-0632. 
W. H. Elashmawi. A Novel Proposed Neural Network MAD (Monitoring, Analysis and Diagnose) Model for Industrial Gas Turbine. International Journal of Soft Computing, 13(3):92-101, 2018. doi:10.36478/ijscomp.2018.92.101.

Encyclopedia Britannica Inc., 1999. Retrieved from: www.britannica.com/technology/gas-turbine-engine.

M. Fast, M. Assadi and S. De. Condition Based Maintenance of Gas Turbines Using Simulation Data and Artificial Neural Network: A Demonstration of Feasibility. Proceedings of ASME Turbo Expo 2008, GT2008-50768, pp. 153-161, Berlin, Germany, 2008. doi:10.1115/gt200850768.

M. Fast, M Assadi and S. De. Development and Multi-Utility of an ANN Model for an Industrial Gas Turbine. Applied Energy, 86(1):9-17, 2009. doi:10.1016/j.apenergy.2008.03.018

M. Fast and T. Palmé. Application of Artificial Neural Network to the Condition Monitoring and Diagnosis of a Combined Heat and Power Plant. Energy, 35(2):1114-1120, 2010. doi:10.1016/j.energy.2009.06.005.

T. Palmé, M. Fast and A. Karlsson. Gas Turbines Sensor Validation through Classification with Artificial Neural Networks, Applied Energy, 88(11):3898-3904, 2011. doi:10.1016/j.apenergy.2011.03.047.

Fortum, and VTT Technical Research Centre of Finland Ltd. 2020. Retrieved from: www.apros.fi/en/.

M. A. Ibrahem, O. Akhrif, H. Moustapha and M. Staniszewski. Neural Networks Modelling of Aeroderivative Gas Turbine Engine: A Comparison Study. Proceedings of the 16th International Conference on Informatics in Control, Automation and Robotics, Vol. 1: ICINCO, pp. 738-745, Prague, Czech Republic, 2019. doi:10.5220/0007928907380745.

F. Jurado. Nonlinear Modelling of Micro-Turbines Using NARX Structures on the Distribution Feeder. Energy Conversion and Management, 46(3):385-401, 2005. doi:10.1016/j.enconman.2004.03.012.

S. Kiakojoori and K. Khorasani. Dynamic Neural Networks for Gas Turbine Engine Degradation Prediction. Health Monitoring and Prognosis. Neural Computing \& Applications, 27(8):2157-2192, 2016. doi:10.1007/s00521015-1990-0.

J. H. Kim, T. W. Song, T. Kim and S. T. Ro. Model Development and Simulation of Transient Behavior of Heavy Duty Gas Turbines. ASME Journal of Engineering for Gas Turbines and Power, 123(3):589-594, 2001. doi:10.1115/1.1370973.

J. H. Kim, T. W. Song, T. Kim and S. T. Ro. Dynamic Simulation of Full Startup Procedure of Heavy-Duty Gas Turbines. ASME Journal of Engineering for Gas Turbines and Power, 124(3):510-516, 2002, doi: 10.1115/1.1473150.

A. Lazzaretto and A. Toffolo. Analytical and Neural Network Models for Gas Turbine Design and Off-Design Simulation. International Journal of Thermodynamics, 4(4):173-182, 2001.

Z. Liu and I. A. Karimi. Gas Turbine Performance Prediction via Machine Learning. Energy, Vol. 192, No. 116627 (10 pages), 2020. doi:10.1016/j.energy.2019.116627.

S. O. T. Ogaji, R. Singh and S. D. Probert. Multiple-Sensor Fault-Diagnosis for a 2-Shaft Stationary Gas Turbine.
Applied Energy, 71(4):321-339, 2002. doi: 10.1016/S03062619(02)00015-6.

A. E. Ruano, P. J. Fleming, C. Teixeiraa, K. R. Vazquezc and C. M. Fonsecaa. Nonlinear Identification of Aircraft Gas Turbine Dynamics. Neurocomputing, 55(3-4):551-579, 2003, doi:10.1016/S0925-2312(03)00393-x.

A. Salehi and M. Montazeri. Black Box Modeling of a Turboshaft Gas Turbine Engine Fuel Control Unit Based on Neural NARX. Journal of Engineering for the Maritime Environment, 233(3):949-956, 2018. doi:10.1177/1475090218797496.

S. Simani and R. Patton. Fault Diagnosis of an Industrial Gas Turbine Prototype Using a System Identification Approach. Control Engineering Practice, 16(7):769-786, 2008. doi:10.1016/j.conengprac.2007.08.009.

Soumalya Sarkar, K. Mukherjee, Soumik Sarkar and A. Ray. Symbolic Transient Time-Series Analysis for Fault Detection in Aircraft Gas Turbine Engines. Proceedings of American Control Conference (ACC), pp. 5132-5137, Montreal, QC, Canada, 2012. doi:10.1109/acc.2012.6315253.

Soumalya Sarkar, K. Mukherjee, Soumik Sarkar and A. Ray. Symbolic Dynamic Analysis of Transient Time-Series for Fault Detection in Gas Turbine Engines. Journal of Dynamic Systems, Measurement, and Control, 135(1): 014506 (6 pages), 2013. doi:10.1115/1.4007699.

K. Suzuki. Artificial Neural Networks - Industrial and Control Engineering Applications. IntechOpen, Croatia, 492 pp., ISBN: 978-953-307-220-3, 2011.

Y. Tiumentsev and M. Egorchev. Neural Network Modeling and Identification of Dynamical Systems. Academic Press, 332 pp., ISBN: 9780128154304, 2019.

G. Torella, F. Gamma and G. Palmesano. Neural Networks for the Study of Gas Turbine Engines Air System. Proceedings of the International Gas Turbine Congress, 8 pages, Tokyo, Japan, 2003.

P. P. Walsh and P. Fletcher. Gas Turbine Performance. Blackwell Science, Osney Mead, Oxford OX2 0EL, UK, 628 pp., 1998. doi:10.1017/S0001924000027834.

Y. Yoru, T. H. Karakoc and A. Hepbasli. Application of Artificial Neural Network (ANN) Method to Exergetic Analyses of Gas Turbines. International Symposium on Heat Transfer in Gas Turbine Systems, 4 pages, Antalya, Turkey, 2009. doi:10.1615/ICHMT.2009.HeatTransfGasTurbSyst.580. 